\title{
CARS Temperature Measurements in a Combustion-Heated Supersonic Jet
}

\author{
S. A. Tedder" \\ College of William and Mary, Williamsburg VA, and NASA Langley Research Center, Hampton VA, 23681 \\ P. M. Danehy ${ }^{\dagger}$ \\ NASA Langley Research Center, Hampton, VA, 23681 \\ and \\ G. Magnotti ${ }^{\ddagger}$ and A. D. Cutler ${ }^{\S}$ \\ The George Washington University, Newport News, VA 23602
}

\begin{abstract}
Measurements were made in a combustion-heated supersonic axi-symmetric free jet from a nozzle with a diameter of $6.35 \mathrm{~cm}$ using dual-pump coherent anti-Stokes Raman spectroscopy (CARS). The resulting mean and standard deviation temperature maps are presented. The temperature results show that the gas temperature on the centerline remains constant for approximately 5 nozzle diameters. As the heated gas mixes with the ambient air further downstream the mean temperature decreases. The standard deviation map shows evidence of the increase of turbulence in the shear layer as the jet proceeds downstream and mixes with the ambient air. The challenges of collecting data in a harsh environment are discussed along with influences to the data. The yield of the data collected is presented and possible improvements to the yield is presented are discussed.
\end{abstract}

\section{Introduction}

$\mathrm{C}$ oherent Anti-Stokes Raman Spectroscopy (CARS) has previously been applied as a non-intrusive diagnostic tool in studies of supersonic combustion. ${ }^{1-8}$ An on-going project at NASA Langley Research Center is using CARS measurements to provide CFD (Computational Fluid Dynamics) modelers with fundamental data sets of the properties of supersonic combustion flows for the improvement of their flow models. Temperature measurements were made with $\mathrm{N}_{2}$ CARS in a ducted supersonic combustion flow. ${ }^{1}$ The same flow was later probed using dualpump CARS and provided not only temperature measurements, but also species concentration of $\mathrm{N}_{2}, \mathrm{O}_{2}$, and $\mathrm{H}_{2}{ }^{9,10}$ This flow was found to be difficult to model computationally and thus a new simpler flow was designed, namely a supersonic free jet with a coaxial injection of fuel $\left(\mathrm{H}_{2}\right)$. Studies of a small-scaled version of this newly designed flow were performed with Dual-Pump CARS and Interferometric Rayleigh Scattering (IRS). ${ }^{11,12}$ IRS was used to measure two components of velocity. The current research effort aims to provide CFD modelers with a simplified flow with additional physical parameters measured simultaneously. The larger scale of the current experiment was used to increase the relative size of the flow length scales compared to the CARS and IRS probe volumes. Several methods from the discipline known as "design of experiments" were used to define the test and defend against experimental measurement systematic errors, to provide meaningful statistics, and to optimize the amount of data taken to the precision desired. The CARS instrument was characterized so that an assessment of the accuracy of the measurements could be made. As part of the experiment, two flow conditions were studied: combusting flow and mixing flow (no fuel). The present paper describes only the mixing flow results, represented by mean maps of the measured quantities with CARS. The IRS results are presented in a separate paper. ${ }^{13}$ Because of the harsh environment of the test facility, the CARS instrument did not always yield reliable data. In this paper the data yield are presented and the effects causing this lowered yield are discussed.

A secondary objective of this paper is to discuss the challenges of applying the Dual-Pump CARS instrument to this larger-scale supersonic free jet to give insight on how these challenges were addressed or suggest how they could be addressed in the future. CARS is a laser-based measurement technique that obtains the Raman spectrum of

\footnotetext{
* Graduate Co-op, Advanced Sensing and Optical Measurement Branch, MS 493, Member AIAA

${ }^{\dagger}$ Research Scientist, Advanced Sensing and Optical Measurement Branch, MS 493, Associate Fellow AIAA

Graduate Student, The George Washington University, Member AIAA

${ }^{\S}$ Professor, The George Washington University, Associate Fellow AIAA
} 
the species present at the crossing of the focal points of three laser beams. The spectra contain temperature and species concentration information. Dual-Pump CARS allows species with Raman shifts in two spectral regions to be overlapped and so increases the number of species measured at once. In this study CARS is applied to a supersonic flow that differs from flows used by previous experimenters. ${ }^{1,2,3,4}$ The flow in this study is a free jet, where flows in previous experiments were ducted. This large-scale free jet flow offers challenges because of the effect on the laser beams traveling long distances through variations in the index of refraction caused by the turbulent flow which has large temperature gradients. This effect on the lasers is referred to as beam steering and can change the laser beam direction and focus which can lower the data yield and increase the standard deviation of the temperature measurements. Another effect that can change the direction and focus of the lasers is the vibration caused by the uncontained supersonic flow on structures and mirrors within the test section. A large temperature variation within the flow created the challenge of having a large dynamic range for the detection of the CARS signal. Other challenges of this study included: the size of the study's measurement space (a $70 \mathrm{~cm}$ axial distance), ambient temperature changes within the test cell, and restricted access to the laser beam relay system during the experiment. The challenge of ambient temperature changes within the test cell was addressed by situating the lasers in a more stable environment outside the test cell. But this removal required a long path length for the lasers presenting additional challenges of maintaining alignment of the laser beams as the beam focus was translated in the flow, and power losses on the multiple mirrors required to reach the test cell.

\section{Test Hardware and Facility}

The experiments were performed in the Direct Connect Supersonic Combustion Facility at NASA Langley Research Center. This facility delivers vitiated air, the hot gas products of $\mathrm{H}_{2}$, air and $\mathrm{O}_{2}$ combustion in which $\mathrm{O}_{2}$ has the same fraction (by mass) as in air. Vitiated air with an enthalpy equivalent to flight at Mach 5.5 was accelerated in a nozzle and discharged into the test cell at atmospheric pressure. The nozzle, described in Ref. 14, created an axisymmetric free-jet flow at Mach 1.6. A drawing of the nozzle is shown on the left of Fig. 1. The heated gas is delivered through the center nozzle with an exit diameter of $6.35 \mathrm{~cm}$, while a concentric cone formed a coflow nozzle that was not used in this experiment reported herein. An infrared image of the heated gas being delivered from the nozzle can be seen on the right side of Fig. 1. Vertical lines in the infrared image are artifacts caused by reflections from the structure holding the optics for the CARS-IRS instrument. The flow rates of gases to the facility heater for the mixing flow condition were: $0.92 \pm 0.012 \mathrm{~kg} / \mathrm{s}$ of air, $0.155 \pm .005 \mathrm{~kg} / \mathrm{s}$ of $\mathrm{O}_{2}$, and

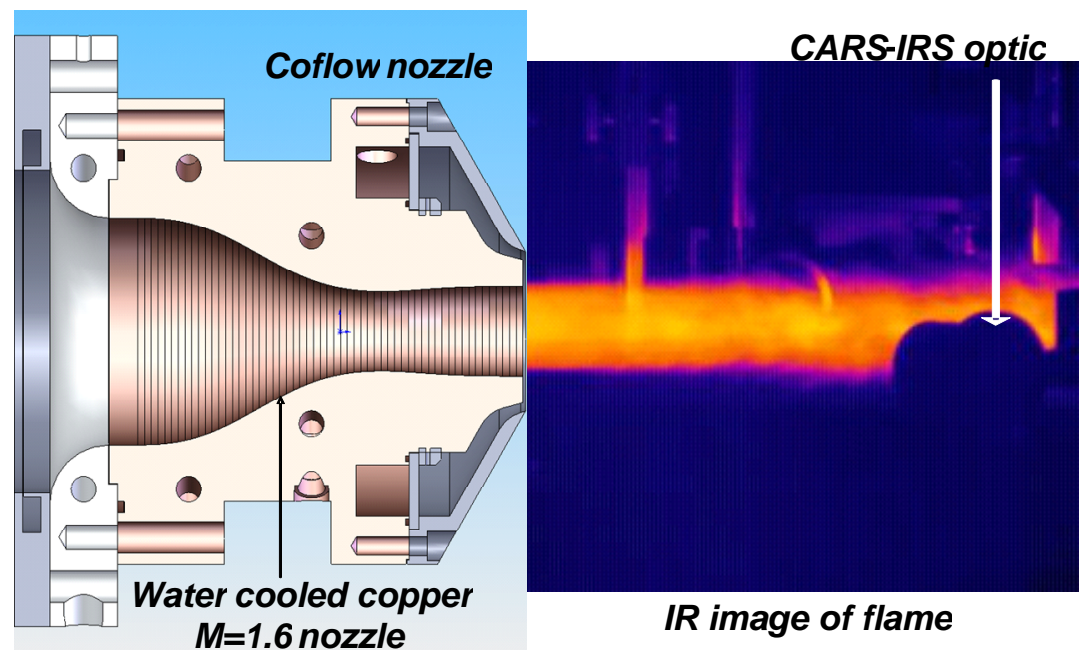

Figure 1. Test apparatus and flow field. Rendering of a section through the model (left hand side) and infrared image of an axi-symmetric free jet for the mixing case (right hand side). Part of the CARS apparatus is shown in the image.

$0.0147 \pm .0004 \mathrm{~kg} / \mathrm{s}$ of $\mathrm{H}_{2}$.

\section{Test Procedure}

To fully characterize the flow field of the axi-symmetric jet, the measurement locations were chosen using design of experiment methods. The locations are shown in Fig. 2, where $(0,0)$ is defined as the geometrical center of the exit of the nozzle and the axial distance increases in the direction of the flow. Because the flow was assumed to be axi-symmetric, most of the locations were chosen in half of a cross section of the flow. Locations outside this half cross section (triangles in Fig. 2) were chosen to check the symmetry of the jet and to assess the alignment of the CARS instrument with respect to the flow. More locations were placed in regions of flow believed to have high gradients in the values being measured, such as the shear layers. Because of a physical limitation of the beam relay 


\section{○ Upstream Locations $\square$ Downstream Locations $\Delta$ Symmetry Check Locations}

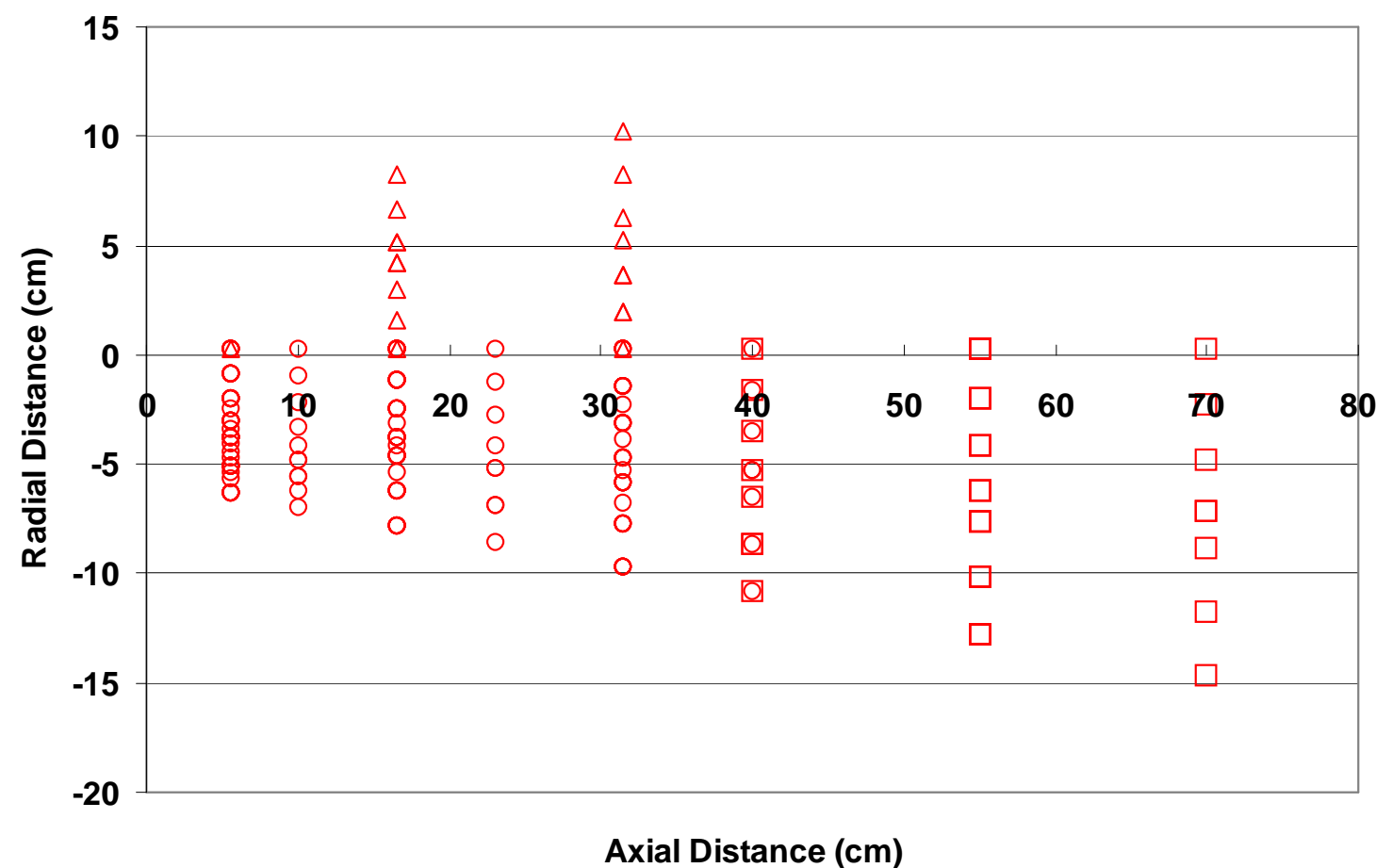

Figure 2. Test matrix used for experiments. Circles indicate the upstream region locations. The symmetrycheck locations are indicated by triangles. The downstream region is indicated by squares. The different measurement locations were visited in random order.

system, the data set was split in two separate regions. These two regions are defined as the upstream region and downstream region are indicated in Fig. 2 by circles and squares, respectively. These two regions overlap at an axial distance of $40 \mathrm{~cm}$. The locations within a given region were visited in a random order so that trends from environmental or instrumental sources would not affect the data set.

The number of samples taken per location was determined by the number required to achieve a pre-determined statistical uncertainty. Every sample for every location could not be taken on the same run. Each run lasted $50 \mathrm{~s}$, limited by the memory of the CARS CCD camera. The $20 \mathrm{~Hz}$ repetition rate of the laser allowed for data collection every $50 \mathrm{~ms}$. Thus, 1000 samples were taken during each run, allowing 4 measurement locations to be visited each run, each having >200 samples per location. These repeated samples at the same location allowed computation of various statistical means, variances, and covariances. Most measurement locations were visited during more than one run to defend against run-to-run trends from environmental or instrumental sources.

\section{CARS Instrumentation}

The Dual-Pump CARS instrument included a mobile cart ${ }^{15}$ was located in a room underneath the test cell to protect the lasers from the large temperature changes and vibrations within the test cell. The cart contained the three lasers required for CARS and IRS. An Nd:YAG laser emitted approximately 1 Joule of energy with a 10 ns pulse duration at a rate of $20 \mathrm{~Hz}$ and at a wavelength of $532 \mathrm{~nm}$. A fraction of the energy from this laser, $250 \mathrm{~mJ}$, pumped a broadband dye laser centered at $604 \mathrm{~nm}$. This red laser had a FWHM of $12 \mathrm{~nm}$ and emits $20 \mathrm{~mJ}$ of energy. Then, $180 \mathrm{~mJ}$ of the energy from the Nd:YAG pumped the narrowband dye laser operating at $552.75 \mathrm{~nm}$. This yellow laser had a FWHM of $0.3 \mathrm{~cm}^{-1}$ and provided an output of $20 \mathrm{~mJ} /$ pulse. Finally, $100 \mathrm{~mJ}$ of the Nd:YAG energy was used as the green beam for CARS and the remaining green energy was used in a pulse stretcher for IRS. More detail of the cart and the Rayleigh instrument can be found in Refs.15, 16, and 17. These three lasers were sent to the test cell though a hole in the test cell floor. In the test cell the lasers were relayed by a series of mostly 3 inch diameter mirrors mounted on a motor-driven beam relay system to the measurement point. This system allowed movement of 
the CARS measurement location in two dimensions, axially and radially, with respect to the flow. For the final two reflections of the beam relay system, 1 inch diameter mirrors were used for each laser separately. In the final reflection the two mirrors directing the red and yellow laser beams were mounted on remotely controlled motorized mounts. These motorized mounts allowed small realignments of the laser beams without entering the test cell which was not accessible during testing. The laser beams, organized in a folded BOXCARS ${ }^{18}$ phase matching geometry, were focused and crossed at their foci with a $40 \mathrm{~cm}$ lens, forming the CARS measurement volume. The measurement volume was measured with a knife edge and found to have the approximate dimensions of 90 micron diameter by $1.5 \mathrm{~mm}$ long. The energies of the lasers when they reach the measurement volume were approximately $15 \mathrm{~mJ}$ in red, $10 \mathrm{~mJ}$ in yellow, and $50 \mathrm{~mJ}$ in green. Energy was lost in the many mirror bounces and other optics as they were passed through the beam relay system from their sources on the cart. The CARS signal created at the measurement volume was collected on the other side of the flow. The signal was then passed through a beam relay system and focused at the exit of a one meter spectrometer with a $2400 \mathrm{line} / \mathrm{mm}$ grating. The slit of the spectrometer was open about $3.4 \mathrm{~mm}$ wide so that the resolution of the spectrum was limited by the size of the focus and not by the aperture of the slit. The slit was opened in this manner to prevent the signal from missing the slit and not entering the spectrometer because of beams steering effects described below. The spectra were taken by a CCD camera with 1340 pixels horizontally and 100 pixels vertically. The vertical direction of the CCD was summed, by groups of 33 pixels, into 3 rows (referred to as bins).

Data taken in the mixing flow of the supersonic jet suffered from motion of the focused signal from shot to shot with respect to the entrance slit of the spectrometer. In Fig. 3 the red dashed lines, labeled as "flow data", show several spectra obtained during a single run in the mixing flow. These flow data are compared in Fig. 3 with spectra taken while there was no mixing flow, shown as blue dotted lines and labeled as "no flow data". The flow data have much greater movement on the CCD camera than the no flow data. There are multiple possible sources of this movement. First, the flow could have caused the structure holding the mirrors and the mirrors themselves to vibrate and change in shape and location. This change of the mirrors and structure is evidenced by the CARS signal moving from its location on the CCD array

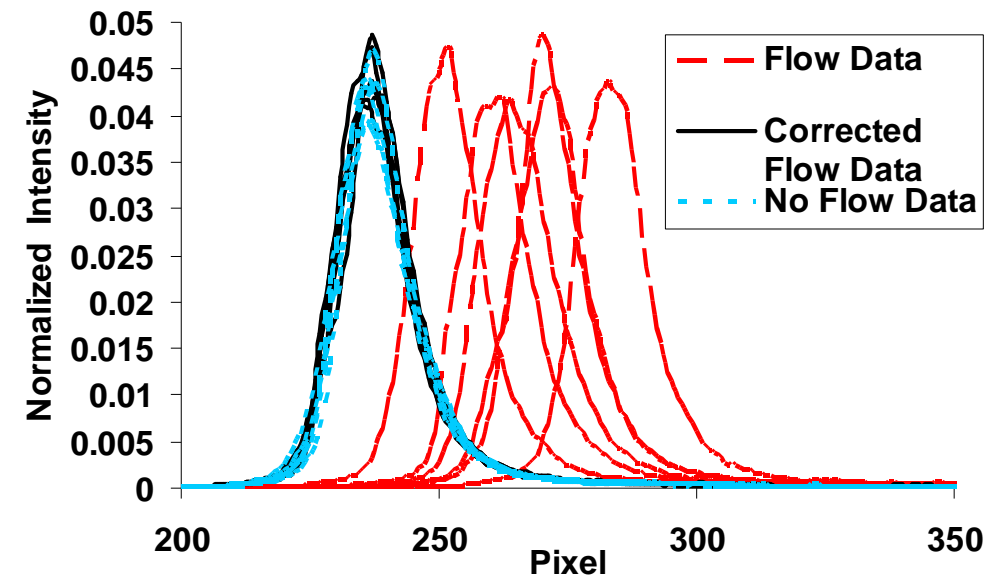

Figure 3. $\mathbf{N}_{2}$ peaks from CARS spectra. $N_{2}$ peaks of CARS spectrum, red dashed lines, taken in supersonic jet at the mixing condition compared with $\mathrm{N}_{2}$ peaks taken as reference data with no flow, dotted blue line, and $N_{2}$ peaks taken in the jet corrected during data analysis.

under no flow conditions to another location while flowing and remaining in this offset position after the flow stopped. Second, a contributing factor to the movement of the signal could have been the temporal and spatial variation of density in the hot, turbulent jet. Since different densities of gas have different indices of refraction, and since a gradient in index of refraction changes the angle of propagation of light, a gradient of density of the gas can change the direction of the beams.

The motion of the CARS signal was reduced by the addition of two 1 meter focal length relay lenses in the path of the CARS signal but still some motion of the signal remained. The vertical motion of the signal created the potential for loss of signal on the CCD array. In defense against this vertical motion the signal was placed into the center of the 3 vertical bins. This allowed motion of the signal both up and down into the other bins without loss of signal. As previously mentioned, after some runs the signal location permanently changed. In this event, the motorized mirror in front of the spectrometer was adjusted to direct the CARS signal back to the center bin. Originally, it was intended to increase the dynamic range of the instrument by dividing the signal over two or more bins. The third bin would be used for background subtraction. However, movement of the signal vertically with respect to the CCD during the runs prevented the use of this approach. Instead, a neutral density filter on a remote switch was added into the signal path to vary the dynamic range of the system. The filter was used in low temperature regions of the flow to prevent saturation of the CCD camera. The filter was removed in hotter parts of 
the flow to improve the signal-to-noise ratio. More details about the structure holding the mirrors (beam relay system) and the instrumental setup up can be found in Ref 15.

\section{CARS Data Analysis}

The CARS data collected in this experiment were analyzed using both in-house written software and a modified version of the widely used Sandia CARSFT code. ${ }^{19}$ Before the spectra were compared to theory in CARSFT, they were processed using in-house codes. The first step was to subtract background light from the spectrum. The background light was either collected in one of the 3 CCD bins where no CARS signal was present or in a spectrum obtained with the red laser beam blocked, preventing the creation of the CARS signal. Next, the spectra were corrected (approximately) for the motion of the focused signal at the entrance to the spectrometer. This correction was made by shifting each spectrum, in wavelength, to match spectra taken while the jet was not operating (no flow data). Using the $\mathrm{N}_{2}$ spectral peak, the spectra were matched at the location in wavelength where $20 \%$ of the spectra's maximum intensity occurred. This location in the spectrum was used because it changes the least in intensity as temperature changes. The results of this shift are shown by black solid lines in Fig. 3. Next, the shape of the broadband dye laser spectrum was removed from the spectra by dividing by a "non-resonant" reference spectrum. Non-resonant reference spectra were obtained by performing CARS in an argon gas cell (which has no resonances in this spectral range) each day of testing prior to the start of testing and again upon the completion of testing. Finally, the square root of the spectral intensities was taken to convert from intensity to susceptibility (for comparison with CARSFT theoretical spectra).

The processed CARS spectra were analyzed using a version of the Sandia code CARSFT that was modified by Lucht et $\mathrm{al}^{20}$ for the use of Dual-Pump CARS and which was further modified by O'Byrne et al. ${ }^{2}$ This code computes theoretical spectra convolved with an instrument function, which is a double Gaussian function optimized to produce a best fit of room temperature theoretical spectra to experiment. The instrument function accounts for the spectral line broadening from several sources, including the finite width of the focus of the CARS signal at the entrance to the spectrometer and the finite resolution of the CCD detector. CARSFT subsequently compares these theoretical spectra to the experimental spectra, iterating on temperature and composition $\left(\mathrm{N}_{2}\right.$ and $\mathrm{O}_{2}$ mole fraction) to minimize an objective function, $\chi^{2} . \quad \chi^{2}$ is a goodness-of-fit parameter calculated using the formula $\sum_{k} \frac{(\text { theory }(k)-\text { data }(k))^{2}}{\operatorname{data}(k)}$, where $\mathrm{k}$ is the pixel number, or wavelength of the spectrum. The theoretical values in this formula have been normalized by setting their maximum value to one. An arbitrary scaling factor is applied to the data for best fit to the theory. Values of $\chi^{2}$ less than one generally produce reasonable fits to the data, judged by eye. Fits to data with $\chi^{2}$ greater than one have large discrepancies by visual inspection.

To provide CFD modelers with the uncertainty in the CARS measurements, the CARS instrument was characterized in a well understood flat flame burner called a Hencken Burner. This type of burner has also been used by Hancock et $\mathrm{al}^{21}$ and others for characterization of CARS systems: data are compared to adiabatic equilibrium calculations of the flame products. The fuel-to-air ratio was varied to obtain a range of temperature and species concentrations. These measurements were made with the CARS system in place in the test cell but the data were not fully analyzed until some time after all the experiments had been completed. The accuracy of the results can be seen in Fig. 4 showing the calculated values of temperature and species concentration compared with means of 1000 CARS measurements. The largest percentage difference of the measured CARS temperature from the calculated temperature is $7.4 \%$ and the average percentage difference is $3.2 \%$. The largest percentage difference of the $\mathrm{O}_{2}$ concentration is $31 \%$ and $\mathrm{N}_{2}$ is $7.2 \%$ percent. The errors in the CARS measurements can be attributed to several possible sources: uncertainty in the flow rates of the gases, spectral overlap of the $\mathrm{N}_{2}$ and $\mathrm{O}_{2}$ spectra, and polarization of the lasers. After the flat flame burner data and mixing flow data were collected, evidence was discovered that the lasers were not all linearly polarized in the same direction, as intended. First the broadband dye laser was observed to be slightly elliptically polarized, when analyzed with a linear polarizer. The CARS signal also showed evidence of elliptical polarization and the orientation of its maximum component of polarization, when measured at the spectrometer, was approximately 45 degrees from the expected direction. For dual-pump CARS a change in polarization of the input beams affects the relative intensity of the spectra for different species, as discussed in Ref. 22. Thus, these elliptical polarizations could cause incorrect measurement of the species concentrations. One standard deviation of the 1000 individual measurements is used as a measure of precision and is shown in the plots in Fig. 4 as error bars. The single-shot precision of temperature averaged over all measured temperatures is $71 \mathrm{~K}$, similar to the value obtained by O'Byrne et al. ${ }^{2}$ In the temperature range (300 to $1000 \mathrm{~K}$ ) for the mixing flow study described below the precision is between $25 \mathrm{~K}$ and $65 \mathrm{~K}$ respectively - similar to Ref. 23. 


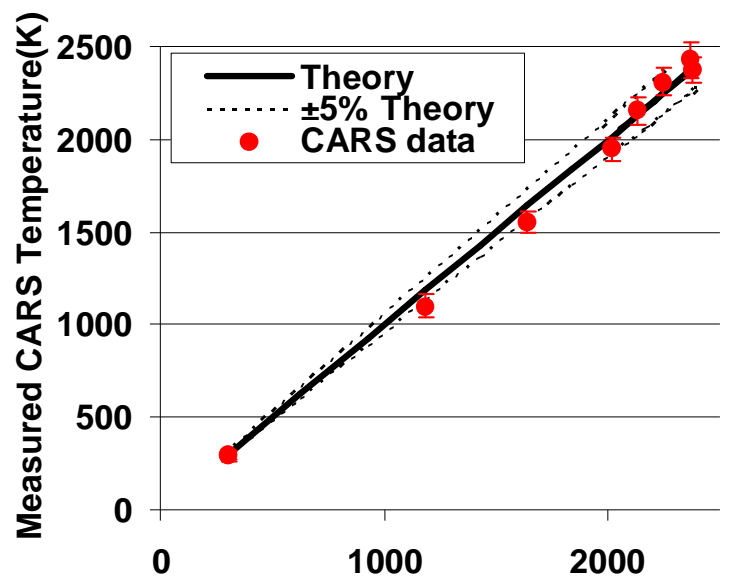

Theoretical Flame Temperature(K)

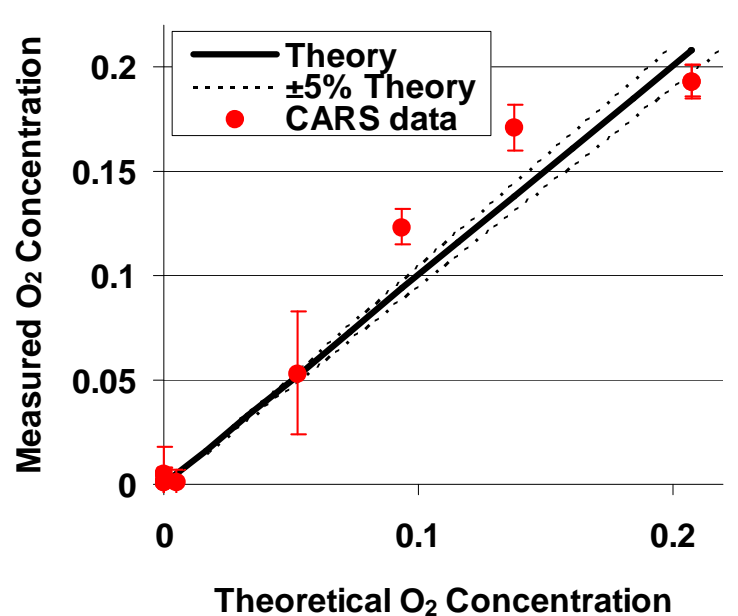

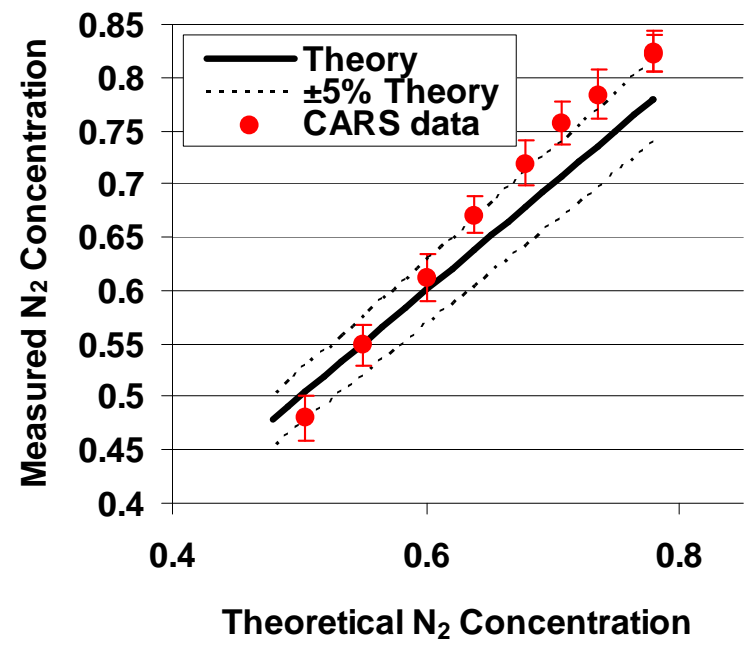

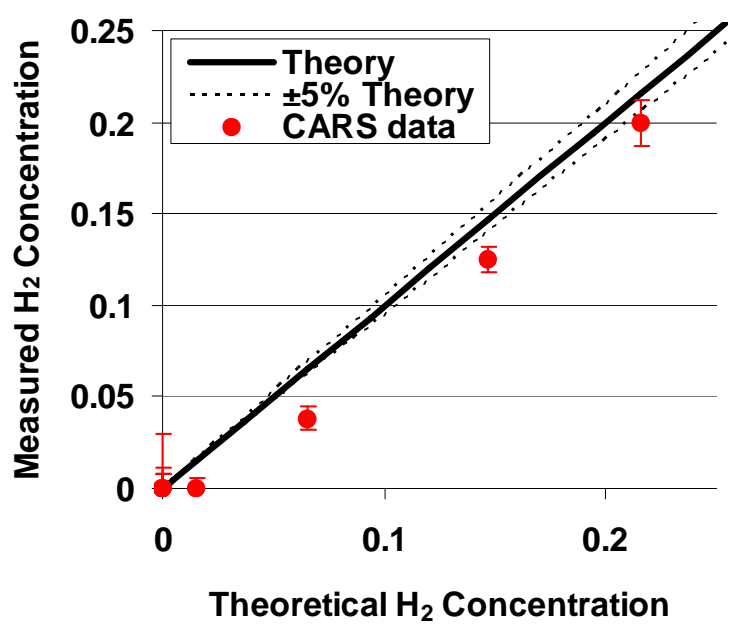

Figure 4: Calculated values versus CARS, measurements taken in flat flame burner with hydrogen fuel. Red dot indicate mean CARS measurements and the error bars indicated the standard deviation. The solid lines show the calculated trends and the dashed lines show 5\% different from the calculated values in the flame burner.

\section{Results and Discussion}

The results of the mixing flow experiment will be presented in this section and will focus on the temperature measurements. Since the errors in the composition measurements were on the same order as the variations in $\mathrm{N}_{2}$ and $\mathrm{O}_{2}$ composition in the flowfield, the composition data are not reported. Figure 5(a) shows the mean of temperature in the form of a contour plot. This plot was generated using the grid of the measured mean values at the locations show in Fig. 2. The temperature is $1010 \pm 49 \mathrm{~K}$ at the first measurement location downstream of the nozzle exit (5.5 $\mathrm{cm})$ and $0.25 \mathrm{~cm}$ from geometric center. The highest temperature gas is indicated by red in the figure. This high temperature starts with the same width as the diameter of the jet $(6.35 \mathrm{~cm})$ and slowly narrows downstream as the heated gas mixes with the ambient air. The mixing layer, in the $500 \mathrm{~K}$ to $700 \mathrm{~K}$ range indicated by green, grows as it moves downstream. The blue color indicates the ambient air temperature of $300 \mathrm{~K}$ and shows the boundary of the jet flow. According to the measurements made in the flat flame burner at $1100 \mathrm{~K}$, the mean temperature measured with CARS may be lower than the actual temperature by $100 \mathrm{~K}$. This indicates that the mean temperatures measured near $1000 \mathrm{~K}$ may be lower than the actual temperature as much as $100 \mathrm{~K}$. Ambient room temperature, $300 \mathrm{~K}$, has a 
higher accuracy. The other temperatures in-between probably have higher accuracy as room temperature is approached.

Figure 5(b) shows a contour plot of the standard deviation of the temperature, in Kelvin. The standard deviation in the plot has been corrected for the CARS instrument single-shot precision. This correction was calculated by taking the difference of the squares of the measured standard deviations and the single-shot precision and then taking the square root of this value. The single-shot precision was measured for temperatures in the flat flame burner, which was assumed to be steady in all flow variables. The single-shot precisions for temperatures not measured in the flat flame burner were found by using a linear interpolation between measured temperatures. In Fig 5 (b), the highest standard deviations of temperature, of about $110 \mathrm{~K}$, are seen in the mixing layer regions. This mixing layer region is shown in the temperature map in Fig. 5(a) as a transition from $950 \mathrm{~K}$ to $650 \mathrm{~K}$. The mixing layer increases in width as it progresses downstream. In the core of the heated flow and in the ambient air, the standard deviation decreases to a range of $50 \mathrm{~K}$ to $70 \mathrm{~K}$ indicating steadier flow conditions.

The standard deviation map in Fig 5(b) shows the combined effect of the unsteadiness of the flow and random errors in the instrument above levels experienced in the quiescent Hencken burner. One such error is related to shotto-shot changes in the width of the CARS spectra caused by changes in the focusing of the CARS signal at the entrance to the spectrometer. Variation in the focus of the CARS spectrum on the CCD changes the width of the instrument function of the detection system. If a constant instrument function width is used in the analysis this

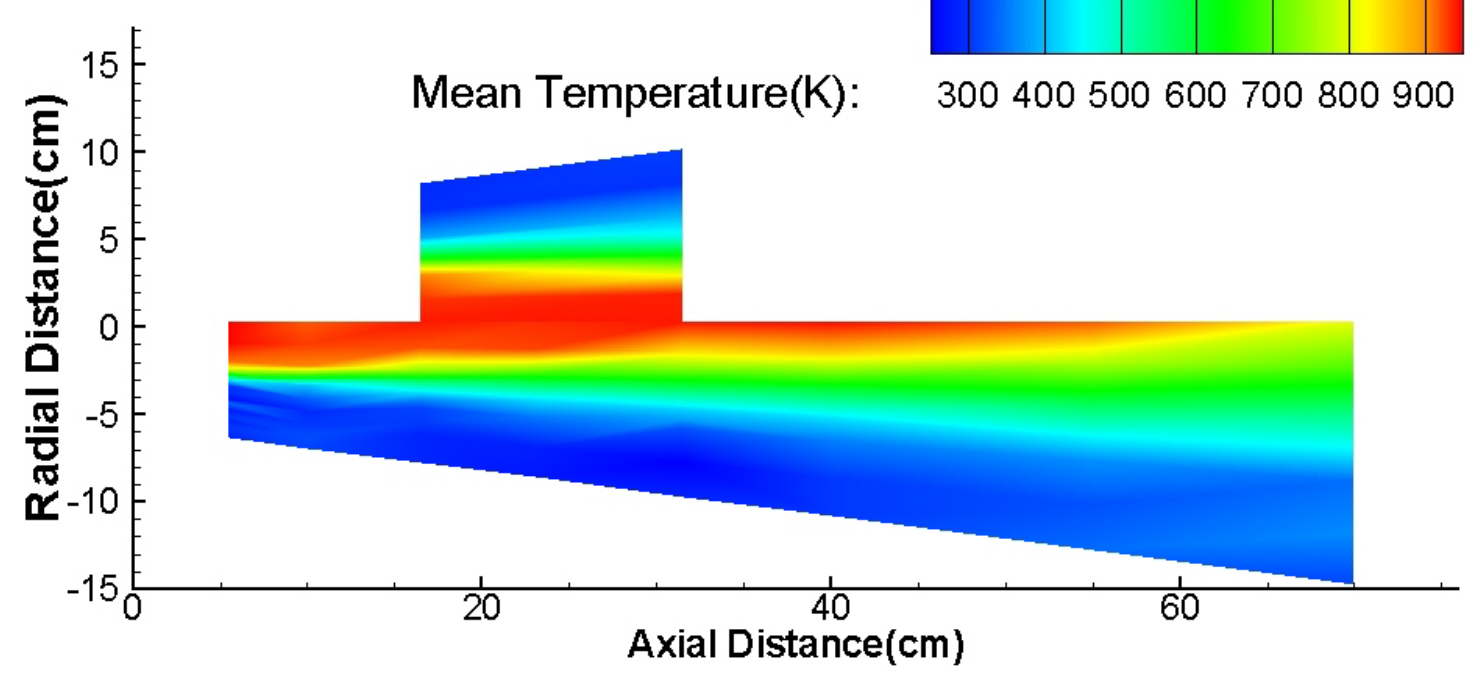

Figure 5(a): Contour map of mean CARS temperature data taken in an axi-symmetric free jet. Radial Distance versus Axial Distance is plotted in centimeters with respect to $(0,0)$ which is the location of the center of the nozzle exit

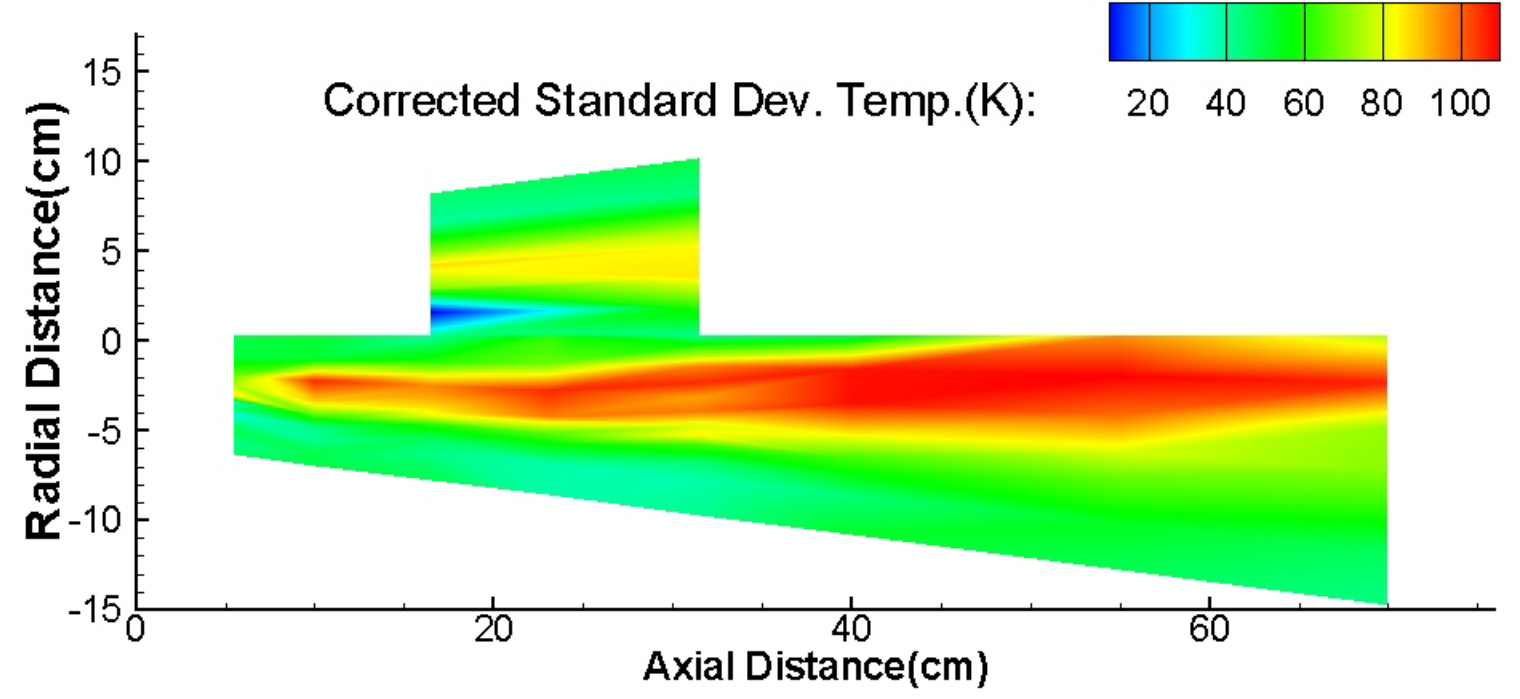

Figure 5(b): Contour map of CARS temperature corrected standard deviation data taken in an axisymmetric free jet. The standard deviation has been corrected by the CARS instrument precision. 
variation in focus leads to erroneous changes in temperature. For the range of temperatures measured in this experiment, $300 \mathrm{~K}-1000 \mathrm{~K}$, the temperatures obtained using CARSFT are mainly determined by the width of the CARS spectra. Fitted temperatures above $1000 \mathrm{~K}$ are sensitive to a combination of the widths of the spectra and the ratio of the intensity of the second vibrational peak to the first vibrational peak. Figure 6 demonstrates shot-to-shot changes in the observed width of the CARS spectra, which lead to erroneous variations in temperature. The spectra in the figure shown with solid lines were taken during operation of the supersonic jet in immediate succession, $50 \mathrm{~ms}$ apart. These spectra were obtained while the jet flow was operating, but with the CARS measurement volume located well outside the jet flow, in the ambient air. The spectra in Fig. 6 vary significantly in width. This width variance is best seen at the half maximum indicated by the arrows in the figure. The variation of the width causes spectra to fit to temperatures both above and below the expected ambient, as indicated in the figure caption. For comparison, a reference CARS spectrum taken with the jet flow off in ambient air is shown in the figure as a dashed black line.

The change in width seen in Fig. 6, while temperature stays relatively constant (room temperature), is caused by a variation in the focus of the CARS signal at the entrance to the spectrometer. This change of focus could be caused by the movement of the location of the

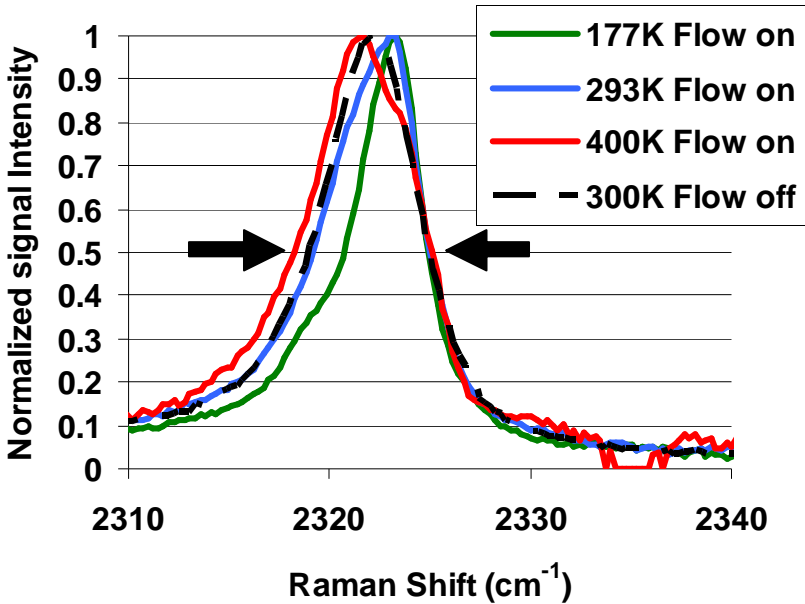

Figure 6: $\mathrm{N}_{2}$ peak of CARS spectra comparing FWHM in ambient air $(300 \mathrm{~K})$ with flow on and off. Normalized signal intensity plotted against frequency (units are wavenumbers) of $\mathrm{N}_{2}$ peaks of CARS signals. Green showing a narrowed $300 \mathrm{~K}$ spectrum fitted to $177 \mathrm{~K}$. Red showing a widen $300 \mathrm{~K}$ spectrum fitted to $400 \mathrm{~K}$. Compared to blue and black fitted to the near room temperature with flow on and of respectively. beams' crossing. If the location of beams' crossing changes, then the distance from where the CARS signal is created to the collimation lens changes. This change in distance to the collimation lens would lead to a change of the focus at the entrance of the spectrometer. Another possible source of change in focus of the CARS signal is the density change in the turbulent gases in the jet as the CARS signal passes through. A density change could change the index of refraction and act like a lens. A lens-like affect could change the collimation of either the input CARS beams or the signal beam, changing the focus at the entrance of the spectrometer.

The effect of a change in the width of the instrument function on the fitted temperature depends on the temperature at which the spectrum was measured. Figure 7 shows the effect of fitting theoretical spectra at various temperatures to theoretical spectra computed assuming a $25 \%$ larger instrument function width. The plot shows that, as the temperature increases, the percentage difference between the fitted temperature and the known temperature decreases. The trend of increasing accuracy at higher temperature is mostly due to the increase in population of the second spectral peak of $\mathrm{N}_{2}$. A bias in the mean temperature to a higher temperature would have occurred if the signal was focused as tightly as possible at the entrance to the spectrometer, since any change in focus during the tests would have increased the instrument function width. In ambient air, with the jet flow off, the CARS signal was slightly defocused allowing both focus and defocus of the signal to occur. Thus, the mean values of the temperatures in ambient air did not show any bias. Unfortunately the change in width of the instrument function is not consistent or predictable on a shot-by-shot basis, because it is caused by random fluctuations in the flow. Therefore standard deviations in the supersonic jet flow

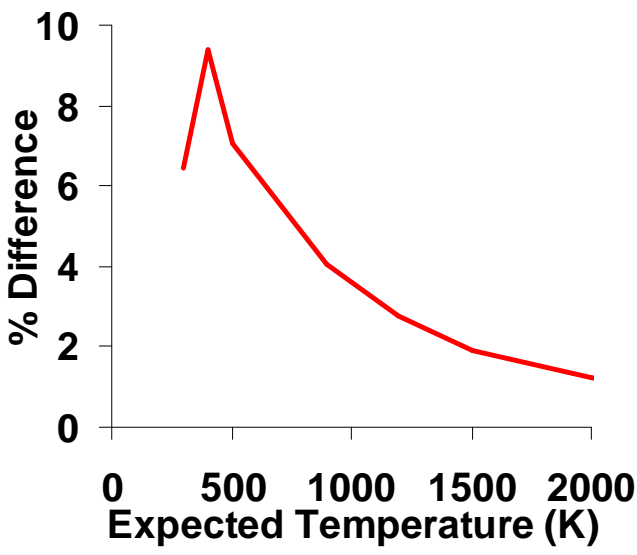

Figure 7: The effect of fitted a CARS spectra with a $25 \%$ larger instrument function width. Percentage difference of the original temperature of the spectrum to the fitting temperature versus the expected temperature. The percentage difference decreases with higher temneratures. 
presented in Fig 5(b) may be overestimated, especially in colder regions of the flow, because, although they are corrected for instrument precision, this correction does not include the effects of change in signal focus at the spectrometer entrance.

Another view of the CARS temperature data is offered in Fig. 8, in which single shot data (data from individual laser pulses) are shown in red. These data points were all taken as a scan during one run. This scan was collected along the axis of the jet for half of the measurement space (the upstream region). The data from the scan are compared with mean values of data acquired with the test matrix, shown as black squares. The error bars in the figure represent the corrected standard deviation for the mean values. The mean values follow the trends of the singleshot spectra shown, for example at the axial distance of $10 \mathrm{~cm}$ where both the mean and the single shot data show a dip in the measured temperature. This dip in temperature may indicate a shock wave or expansion affecting the temperature at this location.

As mentioned in the test procedure section, the data were taken in two regions due to constraints of the beam-relay system. In the one month break between the upstream (including the symmetry check locations) and downstream data set regions minor modifications were made to the facility. These changes raised the measured stagnation pressure in the combustion chamber by nearly $7 \%$, even though the same flow rates were used as before. This change in pressure was accompanied by an increase in temperature measured by CARS: this change can be seen in Fig. 9. The figure compares the mean values of temperature at an axial distance of $40 \mathrm{~cm}$ where the upstream region overlaps the downstream region. The standard deviation at each measurement location is shown by the error bars in the graph. The statistical uncertainties in the means (not shown) are about an order of magnitude smaller than the error bars because these are averages of hundreds of individual measurements. The temperature increase from the downstream region, shown as red squares, compared to the upstream region, shown as black diamonds, is much larger than the statistical uncertainties in the means. This indicates that the change made to the facility changed the properties of the jet flow. Because of this difference in the flow conditions, the two regions should be considered by computational fluid dynamists to be two separate data sets. Note this difference is also present in the plots in Fig. 5, but the data sets have been averaged together at the $40 \mathrm{~cm}$ axial distance.

Table 1 shows the percentage of the data that could not be analyzed because of several factors. These factors are listed in the columns of Table 1 and are detailed below: camera saturation, low signal intensity (below 100 counts), laser-induced breakdown of the gases in the measurement volume, or other factors that resulted in poor fitting to theory. Each factor

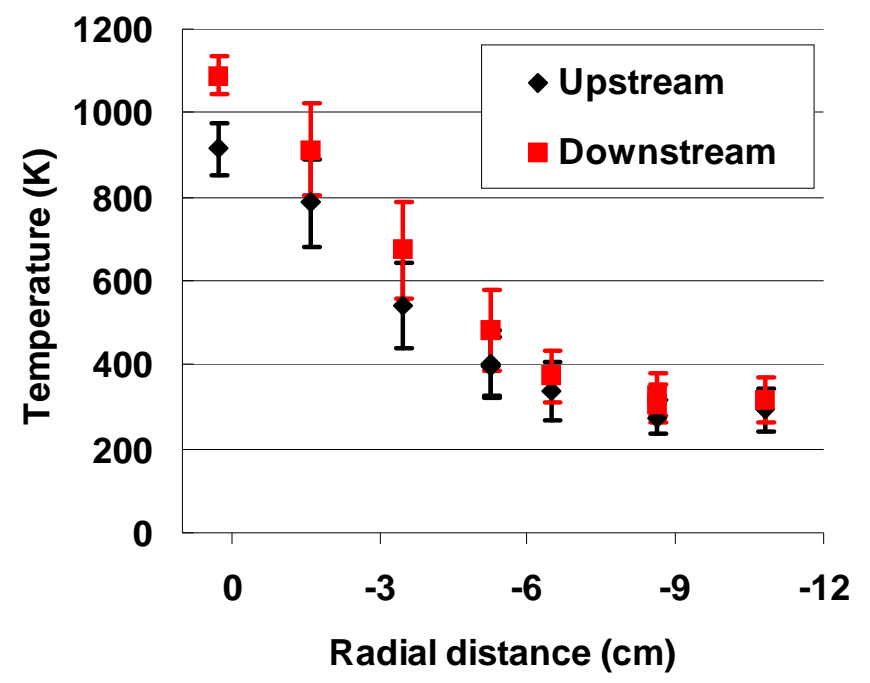

Figure 9: Temperatures taken at the overlap $(40 \mathrm{~cm})$ of the upstream and downstream measurement region. Temperature versus radial distance is plotted. Black diamonds in the plot indicating the measurements taken with the upstream locations and red squares indicating the measurements taken with the downstream locations. Error bars indicate corrected standard deviations. 
contributing to data removal is separated into percentages removed for the upstream region, downstream region, and entire data set.

\begin{tabular}{rccccc} 
& Saturated & Breakdown & Signal $<\mathbf{1 0 0}$ counts & $\chi^{\mathbf{2}}>\mathbf{1}$ (Poor fit) & Yield \\
\cline { 2 - 6 } Upstream & $7.3 \%$ & $0.3 \%$ & $3.3 \%$ & $29.1 \%$ & $60.0 \%$ \\
Downstream & $6.9 \%$ & $0.2 \%$ & $9.1 \%$ & $44.6 \%$ & $39.3 \%$ \\
All Data & $\mathbf{7 . 2 \%}$ & $\mathbf{0 . 2 \%}$ & $\mathbf{4 . 9 \%}$ & $\mathbf{3 3 . 3} \%$ & $\mathbf{5 4 . 4 \%}$
\end{tabular}

Table 1. Percentages removed from data set and total yields. The percentages of data removed for the upstream region, downstream and total data set because of saturation, breakdown, low signal counts, and $\chi^{2}>1$ (poor fit). The last column shows the yields for the data set.

The CCD array of the camera has a saturation threshold. CARS spectra for which a range of pixels exceed that limit are considered saturated. Saturated spectra cannot be fit with the analysis code because a critical part of the spectrum has an unknown intensity. Therefore these data were removed from the data set. The fraction of saturated spectra was the same for upstream and downstream data sets.

Breakdown of the gas sometimes occurs at the location of the beams' crossing. At their crossing, the lasers are focused on top of one another and their combined energies can exceed the threshold of the energy at which the gases or dust particles will dissociate. This dissociation causes a spark or "breakdown". The spark of light is combined with the CARS signal on the CCD array. This effect only contributes to a very small amount of data loss and was the same for upstream and downstream data regions.

Spectra with peak intensities below 100 counts were removed from the data set prior to fitting. The signal-tonoise ratio of these spectra was so low that when the square root of the signal was taken for fitting with CARSFT, it was indistinguishable from the noise. Signal counts below 100 were more frequent in the downstream region indicating an overall lower CARS signal intensity. During the days that the downstream region data was collected, the CARS signal intensity with the flow off was at the same intensity level as the upstream data set. Therefore, the reduction in CARS signal intensity in the downstream region is attributed to effects that occur in response to the flow. Effects that could decrease the CARS signal intensity because of the flow include higher temperatures and increase in turbulence, longer path through turbulence, or more movement of the mirrors. All of these effects would cause more movement of the beams and decrease or eliminate the size of the beams' overlap region. There are two possible reasons that the CARS signal intensity decreased beyond an axial distance of $40 \mathrm{~cm}$. Increased beam steering could have been caused by the natural trends of increased turbulence and larger jet diameter further downstream. Alternately, the instrument or facility could have changed between the measurements. To distinguish between these two possibilities a comparison of the yield at the overlap of the measurement region at an axial distance of $40 \mathrm{~cm}$ was made. The yield for the upstream region is $21 \%$ higher than for the downstream region, even though the measurement location was identical. Along with the change made to the flow facility that increased the temperature in the interim between the measurements of the two regions, a change was also made to the CARS instrumental setup up. This change was the increase of the path length to the measurement volume. This was done by the addition of two mirrors in the path of delivery of the lasers to the measurement volume. These additions could have decreased the stability of the structure and increased the movement of the beams, decreasing the signal level during operation.

Most of the data removed from the data set were from a criterion set on the $\chi^{2}$ value. Removing data that fitted with $\chi^{2}>1$ effectively removed all spectra with peak signal intensities below 1500 counts indicating that the signal-to-noise ratio is too low for these spectra. The requirement of having $\chi^{2}<$ 1 removed more data from the downstream region than the upstream region. This indicates a higher frequency of signal lower that 1500 counts in the downstream region. The decrease in the CARS signal intensity for the downstream data region follows the trend from the "signal below 100 counts" criterion.

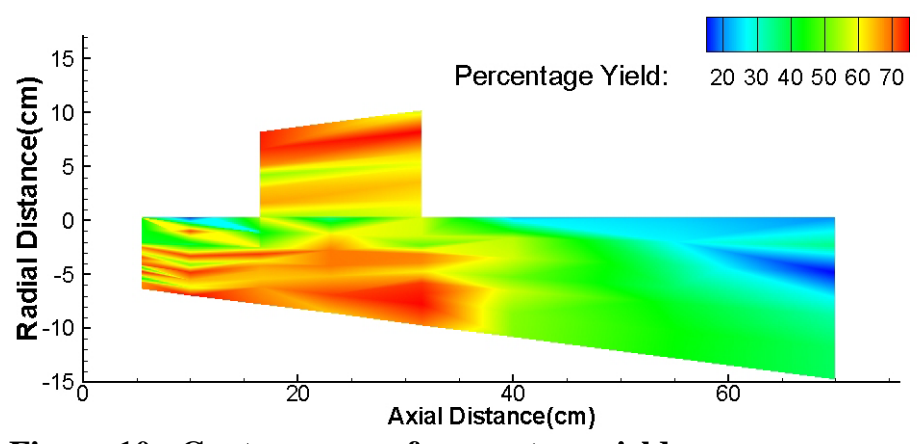

Figure 10. Contour map of percentage yield per measurement location. Yield is dependent on temperature. The upstream region shows more yield than the downstream region. 
The decrease in yield at $40 \mathrm{~cm}$, where the upstream and downstream regions overlap can also be seen in Fig. 10. Figure 10 shows the percentage yield at each measurement location in the form of a contour map. Not only can a sharp drop in the yield be seen between the upstream and downstream data region but also (by comparison with Fig. 5(a)) the effect of temperature on the signal intensity can be seen. The yield decreases at locations in the flow where the temperature is higher. The dependence of the yield on temperature can be attributed to the dependence of the signal intensity (counts) on the temperature as shown in Fig. 11. All the data points used in the results are shown as gray data points in the figure. The data are compared to a theoretical trend of signal intensity versus temperature, as used in Ref. 24, $T^{3.5}$, arbitrarily scaled, is shown as a line in black. The data points generally follow the trend but even at ambient room temperature the signal intensity range is very broad, showing the effect of the beam misalignment to be greater than the temperature effect in most instances. This temperature effect on signal intensity was shown to not create a large bias in the means of temperature in a previous preliminary report on this data set by Danehy et al. ${ }^{25}$

\section{Concluding Remarks}

In conclusion, measurements made with dual-pump CARS in a supersonic symmetric free jet mapped the mean and standard deviation of the temperature field. The data were collected with a yield of $54 \%$. About $7 \%$ of the data were lost because of signal saturation. The majority of the data loss was due to low signal intensity, $38 \%$. This percentage is the addition of the percentage of spectra removed because of signal below 100 counts and the goodness-of-fit criteria of $\chi^{2}>1$. Most of the low-signal data were lost because of CARSFT's inability to distinguish the signal from the noise. Movement of the beams contributed to a change in focus of the CARS signal at the entrance of the spectrometer. This change in focus did not affect the mean values of the temperatures since the CARS signal began slightly defocused so the change in focus was equally likely to increase or decrease the temperature. The change in focus did, however, increase the standard deviations and could not be decoupled from the standard deviation of temperature in the flow. Changes were made to the facility between the measurements of two regions of the flow defined as upstream and downstream. These changes to the facility changed the flow properties of the jet. Because flow conditions for the upstream region and downstream region are different, the regions should be considered separate data sets.

It is recommended to make the following changes to increase the quality of future data sets. To decrease the loss of data from saturation of the CCD, the dynamic range of the CARS instrument needs to be increased. To decrease loss of signal due to beam movement by structural vibration, the beam relay structure needs to be more rigid. To address the loss of signal intensity caused by beam steering by refractive index gradients, the phase matching could be changed to planar BOXCARS, ensuring that at least two beams remain overlapped at the focus. If the spectra were better resolved, then the variation in focus of the CARS signal at the entrance of the spectrometer would affect the temperature less. To improve the accuracy of the CARS concentration measurements, polarizers should be implemented in each input beam, directly before the CARS focusing lens to eliminate any possible elliptical polarization. A polarizer should also be placed in the signal beam before the spectrometer. Also in the experiment, a test case having a larger difference of species (eg. $\mathrm{O}_{2}$ ) from ambient air would provide better distinction between facility air and ambient air. In the future, this data set will be combined with the Rayleigh data set so that the relation of temperature and velocity can be quantified for the benefit of CFD modelers. 


\section{References}

${ }^{1}$ Cutler, A.D., Danehy, P.M., Springer, R.R., O’Byrne, S., Capriotti, D.P., DeLoach, R., "Coherent Anti-Stokes Raman Spectroscopic Thermometry in a Supersonic Combustor," AIAA J., Vol. 41, No. 12, Dec. 2003. pp. 2451-2459.

2 O'Byrne, S., Danehy, P.M., Tedder, S.A., Cutler, A.D., "Dual-Pump Coherent Anti-Stokes Raman Scattering Measurements in a Supersonic Combustor," AIAA J., Vol. 45, No. 4, p. 922-933, April 2007.

${ }^{3}$ Anderson, T. J. and Eckbreth, A. C., "Stimultaneous Coherent Anti-Stokes Raman Spectroscopy Measurements in HydrogenFeuled Supersonic Combustion", J. Propulsion, Vol 8, NO. 1, Jan. 1992.

${ }^{4}$ Eckbreth, A. C., Anderson,T. J., and Dobbs, G. M., "Multi-ColorCARS for Hydrogen-Fueled Scramjet Applications," Applied Physics B, Vol. 45, 1988, pp. 215-223.

${ }^{5}$ Magre, P., Collin, G., Pin, O., Badie, J.M., Olalde, G., Clement, M., "Temperature measurements by CARS and intrusive probe in an air-hydrogen supersonic combustion” Int. Journal of Heat and Mass Transfer, Vol. 44, pp. 4095-4105, 2001.

${ }^{6}$ Smith, M. W., Jarratt, O., Jr., Antcliffe, R. R., Northam, G. B., Cutler, A. D., and Taylor, D. J., "Coherent Anti-Stokes Raman Spectroscopy Temperature Measurements in a Hydrogen-Fueled Supersonic Combustor," Journal of Propulsion and Power, Vol. 9, No. 2, 1993,pp. 163-168.

${ }^{7}$ Yang, S. R., Zhau, J. R., Sung, G. J., and Yu, G., "Multiplex CARS Measurements in Supersonic Hydrogen/Air Combustion," Applied Physics B, Lasers and Optics, Vol. 68, No. 2, 1999, pp. 257-265.

${ }^{8}$ Vereschagin, K. A., Smirnov, V. V., Stelmakh, O. M., Fabelinski, V. I., Sabelnikov, V. A., Ivanov, V. V., Clauss, W., and Oschwald, M., "Temperature Measurements by Coherent Anti-Stokes Raman Spectroscopy in Hydrogen-Fuelled Scramjet Combustor," Aerospace Science and Technology, Vol. 5, No. 5, 2001, pp. 347-355.

${ }^{9}$ O'Byrne, S., Danehy, P. M., and Cutler, A. D., "Dual-Pump CARS Thermometry and Species Concentration Measurements in a Supersonic Combustor," AIAA Paper 2004-0710, Jan. 2004.

${ }^{10}$ Tedder, S., O’Byrne, S., Danehy, P. M., and Cutler, A. D., "CARS Temperature and Species Concentration Measurements in a Supersonic Combustor with Normal Injection,” AIAA Paper 2005-616, 2005.

${ }^{11}$ Bivolaru, D, Danehy, P.M., Grinstead, K.D., Jr., Tedder, S., and Cutler, A.D., "Simultaneous CARS and Interferometric

Rayleigh Scattering," AIAA-2006-2968, 25th AIAA Aerodynamic Measurement Technology and Ground Testing Conference, San Francisco, CA, June 5-8, 2006.

12 Tedder, S., Bivolaru, D., Danehy, P.M., Weikl, M.C., Beyrau, F, Seeger, T., Cutler A. D., "Characterization of a Combined CARS and Interferometric Rayleigh Scattering System”, AIAA Paper 2007-0871, 45 ${ }^{\text {th }}$ AIAA Aerospace Sciences Meeting and Exhibit, Reno, Nevada, January 8-11 (2007)..

${ }^{13}$ Bivolaru D. et al. "Spatially and Temporally Resolved Measurements of Velocity in a $\mathrm{H}_{2}$ - Air Combustion- Heated Supersonic Jet" AIAA Paper 2009-0027, 2009.

${ }^{14}$ Cutler, A.D., Magnotti, G., Baurle, R., Bivolaru, D., Tedder, S., Danehy, P.M., Weikl, M.C., Beyrau, F., and Seeger, T., "Development of Supersonic Combustion Experiments for CFD Modeling," AIAA-2007-0978, 45th AIAA Aerospace Sciences Meeting and Exhibit, Reno, NV, Jan.8-11, 2007.

${ }^{15}$ Bivolaru, D., Lee, J. W., Jones, S. B., Tedder, S., Danehy, P. M., Weikl, M. C., Magnotti, G., Cutler, A. D., "Mobile CARSIRS Instrument for Simultaneous Spectroscopic Measurement of Multiple Properties in Gaseous Flows," 22nd International Congress on Instrumentation in Aerospace Simulation Facilities, Asilomar Conference Center, Pacific Grove, California, June $10-14,2007$

${ }^{16}$ Bivolaru, D., Danehy, P. M., Lee, J. W., Gaffney, Jr. R. L., and Cutler, A. D., "Single-pulse Multi-point Multi-component Interferometric Rayleigh Scattering Velocimeter," AIAA-2006-0836, 44th Aerospace Sciences Meeting, Reno, NV, January 9-12,2006

${ }^{17}$ Bivolaru, D., Danehy, P. M., and Lee, J. W, "Intracavity Rayleigh-Mie Scattering for multipoint, two-component velocity measurement," Optics Letters, Vol. 31, No. 11, pp. 1645-1647, June, 2006.

${ }^{18}$ Eckbreth, A.C., Laser Diagnostics for Combustion Temperature and Species (Gordon \& Breach, Amsterdam, Nederland, 1996).

${ }^{19}$ Palmer, R. E., "The CARSFT Computer Code for Calculating Coherent Anti-Stokes Raman Spectra: User and Programmer Information," Sandia National Laboratories Report SAND89-8206, Livermore, CA,1989.

${ }^{20}$ Lucht, R. P., "Three-Laser Coherent Anti-Stokes Raman Scattering Measurements of Two Species," Optics Letters, Vol. 12, 1987, pp. 78-80.

${ }^{21}$ Hancock, R. D., Bertagnolli, K. E., and Lucht, R. P., "Nitrogen and Hydrogen CARS Temperature Measurements in a NearAdiabatic, Surface- Mixing (Hencken) Burner," Combustion and Flame,Vol.109,1997,pp.323-331.

${ }^{22}$ Hancock, R. D., Schauer, F. R., Lucht, R. P., and Farrow, R. L., "Dual-Pump Coherent Anti-Stokes Raman Scattering (CARS) Measurements of Hydrogen and Oxygen in a Laminar Jet Diffusion Flame," Applied Optics, Vol. 36, 1997, pp. 3217-3226.

${ }^{23}$ O'Byrne, S., Danehy, P. M., and Cutler, A. D., " $\mathrm{N}_{2} / \mathrm{O}_{2} / \mathrm{H}_{2}$ Dual-Pump CARS: Validation Experiments," 20th International Congress on Instrumentation in Aerospace Simulation Facilities, Göttingen, Germany, Aug. 2003.

${ }^{24}$ Danehy, P. M., O’Byrne, S., Cutler, A. D., and Rodriguez, C. G.," Coherent Anti-Stokes Raman Scattering (CARS) as a Probe for Supersonic Hydrogen-Fuel/Air Mixing," Proceedings of the JANNAF APS/CS/PSHS/MSS Joint Meeting [CD-ROM], CPIA, Columbia, MD, Dec. 2003.

25 Danehy, P.M., Magnotti, G., Bivolaru, D., Tedder, S., and Cutler, A.D., "Simultaneous Temperature and Velocity Measurements in a Large-scale, Supersonic, Heated Jet," Paper 1193, 55th JANNAF Propulsion Meeting, Boston, MA, May 12$16,2008$. 